\title{
Caracterização das infrações sanitárias cometidas por estabelecimentos de serviços veterinários no município de Niterói, RJ (2014-2015)
}

[Characterization of health code violations at veterinary establishments in Niteroi, RJ (2014-2015)]

\section{"Artigo Científico/Scientific Article"}

\author{
Flavio Fernando Batista Moutinho ${ }^{1,2 *}$, Cíntia Silva Santos ${ }^{2}$, Maurício Rafael Domingues Rocha ${ }^{2}$, \\ Viviane Moura Azevedo Nunes ${ }^{2}$, Fábio Villas Boas Borges ${ }^{2}$, Francisco Farias Neto ${ }^{2}$
}

\author{
${ }^{1}$ Universidade Federal Fluminense, Faculdade de Veterinária, Niterói-RJ, Brasil. \\ ${ }^{2}$ Fundação Municipal de Saúde / DEVIC / Centro de Controle de Zoonoses, Niterói-RJ, Brasil. \\ *Autor para correspondência/Corresponding author: E-mail: flaviomoutinho@id.uff.br
}

\begin{abstract}
Resumo
O objetivo do presente trabalho foi caracterizar as infrações sanitárias cometidas por estabelecimentos de serviços veterinários (consultórios, clínicas, serviços de diagnóstico e pet shops) em Niterói, RJ, no período 2014-2015. Trata-se de estudo retrospectivo com base em levantamento documental efetuado nos registros de autos de infração lavrados pelos fiscais sanitários da Vigilância Sanitária. Dos 122 autos de infração lavrados contra estabelecimentos de serviços veterinários no período, houve predomínio daqueles contra pet-shops ( $n=84 / 68,9 \%)$, seguido de consultórios veterinários $(n=27 / 22,1 \%)$, clínicas $(n=10 / 8,2 \%)$ e serviço especializado $(\mathrm{n}=1 / 0,8 \%)$. O principal motivo da lavratura foi o descumprimento de Termo de Intimação $(n=73 / 59,8 \%)$, seguido de falta de licenciamento sanitário $(n=48 / 39,3 \%)$ e ambos os motivos simultaneamente $(\mathrm{n}=1 / 0,9 \%)$. O estudo permite concluir que o descumprimento da legislação sanitária é contumaz em todos os tipos de estabelecimentos veterinários fiscalizados. As causas que vêm motivando a lavratura contra esses estabelecimentos são o descumprimento de Termos de Intimação e a falta de licenciamento sanitário. Os resultados reforçam a necessidade de desenvolvimento de ações de educação continuada junto aos responsáveis pelos estabelecimentos visando sensibilizá-los para a importância e a necessidade de cumprimento da legislação sanitária.
\end{abstract}

Palavras-chave: Vigilância sanitária; serviços veterinários; saúde animal.

\begin{abstract}
The objective of the current project was to characterize the health offenses committed by veterinary establishments located in Niteroi, RJ, in the period between 2014 and 2015. This is a retrospective study based on a document survey on infraction notices issued by the health surveillance. From the 122 infraction notices issued against veterinary establishments during this period, there was a strong predominance of the ones against pet shops $(n=84 / 68.9 \%)$, followed by the ones against veterinary practices $(n=27 / 22.1 \%)$, clinics $(n=10 / 8.2 \%)$, and specialized services $(n=1 / 0.8 \%)$. The main reason for the issuance of the infraction notices was noncompliance with the Intimation Terms (IT) $(n=73 / 59.8 \%)$, followed by the lack of sanitary licensing $(\mathrm{n}=48 / 39.3 \%)$, and both reasons simultaneously $(\mathrm{n}=1 / 0.9 \%)$. Due to the current study, it was possible to conclude that the failure to comply with sanitary laws is customary in all types of establishments. The problems that have been causing the issuance of infraction notices against these establishments are the noncompliance with Intimation Terms and the lack of sanitary licensing. The results reinforce the need to develop actions of continued education of the people responsible for the veterinary establishments, with the intention to raise awareness of the importance and necessity of compliance with the current sanitary law.
\end{abstract}

Keywords: Health surveillance; veterinary service; animal health. 


\section{Introdução}

Os estabelecimentos de serviços veterinários incluem aqueles em que são desenvolvidos procedimentos de competência exclusiva dos médicos veterinários, como consultórios, ambulatórios, clínicas, laboratórios e hospitais veterinários; e, também, os de interesse veterinário, que são aqueles em que pode ser exigida a presença do responsável técnico médico veterinário, em função do tipo de atividade realizada, como no caso de hotéis para animais, pet-shop com e sem banho e tosa, salão de banho e tosa e canis (ANVISA, 2010).

O Conselho Federal de Medicina Veterinária (CFMV) conceitua e estabelece condições para o funcionamento de consultórios, ambulatórios, clínicas e hospitais veterinários (CFMV, 2012) e o Conselho Regional de Medicina Veterinária do estado do Rio de Janeiro complementa essas exigências em nível estadual (CRMV-RJ, 2012) e conceitua e estabelece condições para o funcionamento dos serviços veterinários especializados, que incluem, dentre outras atividades, àquelas referentes aos laboratórios de diagnóstico e os estabelecimentos de diagnóstico por imagem (CRMV-RJ, 2014). Além disso, o CFMV regulamenta o funcionamento dos petshops com e sem serviço de estética para animais (CFMV, 2010).

A atuação da vigilância sanitária em relação aos estabelecimentos de serviços veterinários ainda traz uma série de divergência de entendimentos e interpretações em relação às competências legais para a fiscalização, especialmente no que diz respeito à questão da estrutura física dos estabelecimentos. Nesse contexto, essa atuação vem sendo feita de maneira muito diferenciada entre os municípios brasileiros (ANVISA, 2010).

Em 2010, a Secretaria de Estado de Saúde e Defesa Civil do Rio de Janeiro descentralizou para os municípios fluminenses a competência para a concessão, revalidação e cancelamento de licença sanitária dos estabelecimentos de serviços veterinários (Rio de Janeiro, 2010). Apesar disso, o município de Niterói, através do Departamento de Vigilância Sanitária e Controle de Zoonoses (Devic) já efetuava tal fiscalização desde 2008, já que ela está prevista no Código Sanitário do município (Niterói, 2008).

A ANVISA (2010) entende, em relação aos estabelecimentos de serviços veterinários, que à vigilância sanitária compete, principalmente, a fiscalização de fatores que envolvam a prevenção de riscos e agravos à saúde humana, englobando a questão da saúde ocupacional, gerenciamento de resíduos, abastecimento de água, radiações ionizantes, higiene e limpeza e medicamentos da linha humana.

No município de Niterói, durante o processo fiscalizatório os fiscais verificam principalmente, com fulcro no conceito de risco sanitário, as condições estruturais dos estabelecimentos, no que diz respeito à infraestrutura mínima necessária e seu estado de conservação; as condições higiênicosanitárias dos locais e dos animais porventura existentes; os produtos utilizados e ou comercializados, no que diz respeito à sua legalidade, conservação, acondicionamento e prazo de validade; os documentos obrigatórios, como comprovante de desinsetização e desratização e laudo que ateste a qualidade da água de abastecimento, dentre outros.

O objetivo do presente trabalho foi caracterizar as infrações sanitárias cometidas por estabelecimentos veterinários localizados no município de Niterói, RJ, no período 2014-2015, em função da tipificação da infração e do tipo de estabelecimento infrator.

\section{Material e Métodos}

O município de Niterói integra a Região Metropolitana do Rio de Janeiro, limitando-se com os municípios de São Gonçalo e Maricá, a baía da Guanabara e o oceano Atlântico (TCE-RJ, 2013). Com população estimada para 2015 de 496.696 habitantes (IBGE, 2015), o município está dividido em cinco regiões de planejamento, as quais contemplam 52 bairros.

No presente estudo retrospectivo foi efetuado um levantamento documental nos arquivos da Seção de Controle de População Animal, do Centro de Controle de Zoonoses (CCZ) da Prefeitura de Niterói, RJ, Brasil. O CCZ integra o Departamento de Vigilância Sanitária e Controle de Zoonoses (DEVIC), da Fundação Municipal de Saúde do município e a referida Seção é responsável pelo licenciamento e fiscalização de estabelecimentos de serviços veterinários no município. Estão incluídos no universo passível de fiscalização, os consultórios, ambulatórios, clínicas, hospitais, serviços de diagnóstico e pet shops. No período do estudo, existiam 183 estabelecimentos de serviços veterinários cadastrados na Vigilância Sanitária Municipal de Niterói, sendo 114 pet-shops, 45 consultórios, 18 
clínicas e seis serviços especializados (laboratórios e estabelecimentos de diagnóstico por imagem). Não havia no município ambulatórios e hospitais veterinários, logo, eles não foram incluídos na análise.

O referido levantamento documental envolveu todos os Autos de Infração lavrados pela vigilância sanitária municipal contra estabelecimentos de serviços veterinários, entre janeiro de 2014 e dezembro de 2015.

Os dados obtidos foram tabulados $\mathrm{e}$ analisados com técnicas de estatística descritiva, utilizando-se o programa Exce ${ }^{\circledR}$ para o cálculo das frequências absoluta e relativa de Autos de Infração lavrados no período, em função do tipo de infração e do tipo de estabelecimento.

\section{Resultados e Discussão}

Dos 122 autos de infração lavrados contra estabelecimentos veterinários no período avaliado, houve forte predomínio daqueles contra os petshops $(68,9 \% / n=84)$, seguido dos lavrados contra os consultórios veterinários $(22,1 \% / \mathrm{n}=27)$, clínicas $(8,2 \% / \mathrm{n}=10)$ e serviço especializado/laboratório $(0,8 \% / \mathrm{n}=1)$.

Quando se leva em consideração a quantidade de Autos de Infração lavrados por tipo de estabelecimento, em relação à quantidade cadastrada no Devic de cada tipo, essa ordem se mantém, apesar de variar a proporção, podendo-se perceber que, proporcionalmente, o percentual de consultórios veterinários que foram infracionados $(53,3 \%)$ é maior que o de pet-shops $(51,7 \%)$, como pode ser visto na Tabela 1.

Tabela 1. Proporção de Autos de Infração lavrados em relação ao número de estabelecimentos veterinários cadastrados no Centro de Controle de Zoonoses. Niterói, RJ,2014-2015.

\begin{tabular}{lllll}
\hline $\begin{array}{c}\text { Tipo de } \\
\text { estabelecimento }\end{array}$ & $\begin{array}{c}\text { Quantidade } \\
\text { cadastrada }\end{array}$ & $\begin{array}{c}\text { Quantidade } \\
\text { infracionada }\end{array}$ & $\begin{array}{c}\text { Percentual de } \\
\text { Estabelecimentos } \\
\text { Infracionados }\end{array}$ & $\begin{array}{c}\text { Número de Autos de } \\
\text { Infração lavrados }\end{array}$ \\
\hline Pet-shop & 114 & 59 & 51,7 & 84 \\
Consultório & 45 & 24 & 53,3 & 27 \\
Clínica & 18 & 06 & 33,3 & 10 \\
Serviços & 6 & 01 & 16,7 & 01 \\
especializados & & $\mathbf{4 9 , 2}$ & $\mathbf{1 2 2}$ \\
\hline Total & $\mathbf{1 8 3}$ & $\mathbf{9 0}$ & $\mathbf{4 9 , 2}$ & \\
\hline
\end{tabular}

O principal motivo da lavratura de auto de infração foi o descumprimento de Termo de Intimação $(59,8 \% / \mathrm{n}=73)$, seguido de falta de licenciamento sanitário $(39,3 \% / \mathrm{n}=48)$ e ambos os motivos simultaneamente $(0,9 \% / \mathrm{n}=1)$.

No caso dos pet-shops $(61,9 \%)$ e consultórios $(59,2 \%)$, houve predomínio da infração de descumprimento de Termo de Intimação; no caso das clínicas houve a mesma quantidade de infrações por descumprimento de TI e para falta de licenciamento sanitário; já nos serviços veterinários só houve uma infração, no caso por falta de licenciamento sanitário de um laboratório (Figura 01).

A infração por falta de licenciamento sanitário (inicial ou revalidação) ocorreu em 27\% pet-shops, $28 \%$ das clínicas, $24 \%$ dos consultórios e $17 \%$ dos serviços especializados. De acordo com o Código Sanitário de Niterói (Niterói, 2008), é considerada infração leve, o estabelecimento não requerer o licenciamento inicial ou anual no prazo estabelecido, sujeitando o infrator às penalidades de advertência, interdição, cancelamento de autorização e licença, intervenção e/ou multa.

Todos os estabelecimentos de proteção e interesse à saúde, pessoa física ou jurídica, devem requerer a licença sanitária inicial antes de entrar em funcionamento e a mesma deve ser revalidada anualmente (Niterói, 2008) e, de acordo com a Secretaria de Estado de Saúde do Rio de Janeiro, é atribuição da Secretaria Municipal de Saúde inspecionar os estabelecimentos antes da concessão e revalidação da licença sanitária (Rio de Janeiro, 2010). O CRMV-RJ também considera a Licença Sanitária uma condição para o funcionamento dos estabelecimentos veterinários no estado (CRMV-RJ, 2012; CRMV-RJ, 2014). Cabe ressaltar que o licenciamento não deve ser considerado mera formalidade burocrática. Ele protege a população de modo geral, ao ajudar na 
mitigação dos riscos sanitários advindos das atividades licenciadas e, ao mesmo tempo, protege o setor regulado, ao possibilitar que o mesmo esteja devidamente regularizado junto ao órgão sanitário.

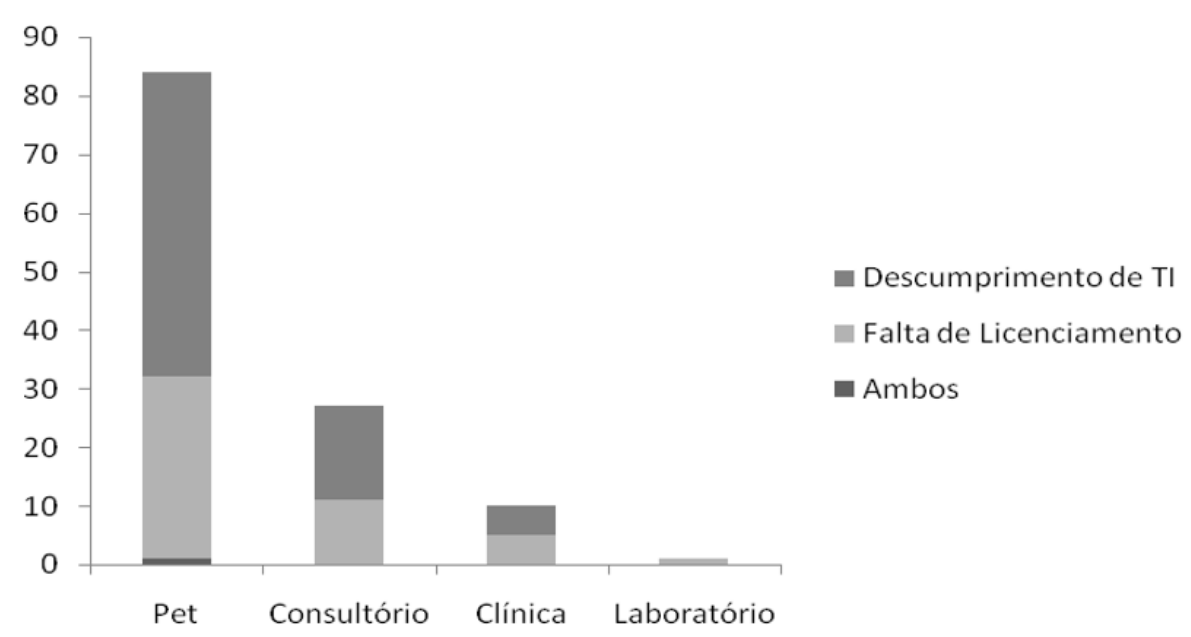

Figura 1. Quantidade de Autos de Infração lavrados, por tipo de estabelecimento e tipo de infração, em Niterói, RJ, 2014-2015.

Houve infração de descumprimento de Termo de Intimação em $46 \%$ dos pet-shops, $36 \%$ dos consultórios e $28 \%$ das clínicas. Essa infração não foi constatada em nenhum serviço especializado. $\mathrm{O}$ descumprimento de exigência de Termo de Intimação no prazo concedido é considerado infração grave, sujeitando o infrator às penalidades de multa e/ou interdição total ou parcial do estabelecimento e cassação da licença sanitária (Niterói, 2008).

No processo de fiscalização são verificadas as condições higiênico-sanitárias e de conservação do estabelecimento, além da documentação apresentada. No caso da detecção de não conformidades é lavrado um Termo de Intimação com um prazo para que o estabelecimento se adeque às exigências $\mathrm{e}$, por conseguinte, à legislação sanitária vigente (Niterói, 2008; ANVISA, 2010).

Para Labarthe e Pereira (2008), o descumprimento de regras básicas envolvendo a segurança sanitária coletiva e individual é contumaz na clínica veterinária de pequenos animais e tal fato é creditado ao desconhecimento desses profissionais e não à indisciplina ou negligência. Nesse contexto, as autoras destacam a necessidade de educação continuada para esses profissionais. Estudo desenvolvido em Ribeirão Preto, SP, mostrou que os estabelecimentos veterinários apresentavam condições inadequadas de biossegurança, em especial no que diz respeito ao gerenciamento de resíduos, proteção radiológica, saúde e segurança do trabalhador e armazenamento e manutenção dos produtos veterinários (Valente, 2004).

Importante ressaltar que, a despeito de toda a discussão atual sobre a questão da saúde única e considerando os riscos sanitários que os estabelecimentos veterinários podem oferecer para a saúde humana e animal, a quantidade de artigos publicados envolvendo a questão da vigilância sanitária de estabelecimentos veterinários é baixíssima o que acaba por prejudicar uma discussão mais aprofundada dos resultados encontrados.

\section{Conclusão}

$\mathrm{O}$ presente estudo permite concluir que o descumprimento da legislação sanitária em estabelecimentos veterinários de Niterói, RJ, foi contumaz em todos os tipos de estabelecimentos veterinários que foram fiscalizados no período avaliado.

As causas que motivaram a lavratura de Autos de Infração contra esses estabelecimentos foram o descumprimento de Termos de Intimação e a falta de licenciamento sanitário. A despeito do Código Sanitário Municipal ter sido promulgado há oito anos, os resultados reforçam a necessidade de desenvolvimento de ações de educação continuada junto aos responsáveis pelos estabelecimentos veterinários localizados no município visando a sensibilizá-los para a importância e a necessidade de cumprimento da legislação sanitária vigente.

Nesses estabelecimentos, além do risco de transmissão de zoonoses, há risco de transmissão de doenças para outros animais e riscos inerentes à 
saúde do trabalhador, como no uso de medicamentos e de radiação ionizante. A saúde animal pode refletir direta ou indiretamente na saúde humana, portanto, a vigilância sanitária tem papel crucial para que os estabelecimentos de saúde animal cumpram a legislação sanitária minimizando o risco à saúde humana e, também, dos outros animais.

Por fim, cabe ressaltar a necessidade e importância do incremento das pesquisas na área da vigilância sanitária de estabelecimentos veterinários no Brasil.

\section{Conflito de Interesse}

Os autores declaram não existir conflito de interesse.

\section{Referências}

ANVISA. Referência técnica para o funcionamento dos serviços veterinários. Brasília: ANVISA, 2010.

CONSELHO FEDERAL DE MEDICINA VETERINÁRIA - CFMV. Resolução $\mathbf{n}^{\circ}$ 1015, de 09 de novembro de 2012. Conceitua e estabelece condições para o funcionamento de estabelecimentos médico-veterinários de atendimento a pequenos animais e dá outras providências. Disponível em: $<$ http://portal.cfmv.gov.br/portal/lei/index/id/ 441>. Acesso em: 08 jun. 2016.

CONSELHO REGIONAL DE MEDICINA VETERINÁRIA DO ESTADO DO RIO DE JANEIRO - CRMV- RJ. Resolução no 27, de 16 de maio de 2012. Dispõe sobre o funcionamento dos estabelecimentos Médicos Veterinários no âmbito do Estado do Rio de Janeiro. Disponível em: <http://www.crmvrj.org.br/Resolucao272012 .doc>. Acesso em: 08 jun. 2016.

CONSELHO REGIONAL DE MEDICINA VETERINÁRIA DO ESTADO DO RIO DE JANEIRO - CRMV - RJ. Resolução no 041 de 01 de julho de 2014. Dispõe sobre a instalação e o funcionamento dos Serviços de Veterinária Especializados no âmbito do Estado do Rio de Janeiro. Disponível em: http://www.crmvrj.org.br/wpcontent/uploads/2014/07/Resolu\%C3\%A7\% C3\%A3o-CRMV-RJ-041-2014.pdf. Acesso em: 08 jun. 2016.
CONSELHO FEDERAL DE MEDICINA VETERINÁRIA - CFMV. Resolução $\mathbf{n}^{\circ}$ 1060, de 27 de outubro de 2014. Dispõe sobre Diretrizes Gerais de Responsabilidade Técnica em estabelecimentos comerciais de exposição, manutenção, higiene estética e venda ou doação de animais, e dá outras providências. Disponível em: <http://portal.cfmv.gov.br/portal/lei/index/id/ 454>. Acesso em: 08 jun. 2016.

RIO DE JANEIRO. Resolução SESDEC n 1411, de 15 de outubro de 2010. Delega competências de ações de vigilância sanitária para as secretarias municipais de saúde no âmbito do estado do rio de janeiro e dá outras providências. Disponível em: <http://www.legislacaodesaude.rj.gov.br/com ponent/content/article/9-resolucoes/5188resolucao-sesdec-n14112010.html?highlight=WzE0MTFd\&Ite mid=235>. Acesso em: 06 jun. 2016.

NITERÓI. Lei no 2564, de 25 de junho de 2008. Dispõe sobre o Código Sanitário do Município de Niterói. Disponível em: <http://www.fazenda.niteroi.rj.gov.br/inetpub /wwwroot/smf_pmn/www/catnit/030L08002 564.pdf>. Acesso em: 06 jun. 2016.

TRIBUNAL DE CONTAS DO ESTADO DO RIO DE JANEIRO - TCE-RJ. Estudos socioeconômicos dos municípios do Estado do Rio de Janeiro 2013: Niterói. Rio de Janeiro: TCE/SGP, 2013.

INSTITUTO BRASILEIRO DE GEOGRAFIA E ESTATÍSTICA. Estimativa da população 2015. Disponível em: < ftp://ftp.ibge.gov.br/Estimativas_de_Populac ao/Estimativas_2015/estimativa_2015_TCU_ 20160712.pdfftp://ftp.ibge.gov.br/Estimativa s_de_Populacao/Estimativas_2015/estimativa _2015_TCU_20160712.pdf>. Acesso: 09 set. 2016.

Labarthe, N.; Pereira, ME.C. Biossegurança na experimentação e na clínica veterinária de pequenos animais. Ciência Veterinária nos Trópicos, 11(1):153-157,2008.

Valente, D.; Oliveira, C.A.A.; Rodrigues, V.C.; Trebbi, H.. Biossegurança em estabelecimentos veterinários. Revista de Educação Continuada em Medicina Veterinária e Zootecnia do CRMV-SP, 7(1/3):45-54, 2004. 